\title{
Sigmoid Colon Adenocarcinoma with Isolated Loss of PMS2 Presenting in a Patient with Synchronous Prostate Cancer with Intact MMR: Diagnosis and Analysis of the Family Pedigree
}

\author{
WEIDONG LIU ${ }^{1}$, DONGWEI ZHANG ${ }^{2}$, SANDA A. TAN ${ }^{3}$, XIULI LIU ${ }^{2}$ and JINPING LAI ${ }^{2}$ \\ ${ }^{1}$ Department of Surgery, Xiangya Hospital, XiangYa School of Medicine, \\ Central South University, Changsha, P.R. China; \\ ${ }^{2}$ Department of Pathology, Immunology and Laboratory Medicine, \\ University of Florida College of Medicine, Gainesville, FL, U.S.A.; \\ ${ }^{3}$ Department of Surgery, University of Florida College of Medicine, Gainesville, FL, U.S.A.
}

\begin{abstract}
Lynch syndrome (LS) patients with isolated PMS2 loss in the colon cancer, while intact MMR in the prostate cancer, are exceedingly rare. Herein, we report such a case. A 71-year-old male was found to have increased serum PSA $(10 \mathrm{ng} / \mathrm{ml})$ after treatment for his urinary tract infection. Prostate biopsies showed foci of prostate cancer with Gleason score $7(3+4)$ (grade grope 2) involving 10\% of two cores. Through work up for treatment of the prostate cancer, he was found to have focal thickening of his sigmoid colon with adjacent lymphadenopathy in CT scans. Colon biopsy showed a tubular adenoma with high-grade dysplasia and deep invasive carcinoma could not be excluded. A low anterior resection of the rectosigmoid colon was performed and a sigmoid colon adenocarcinoma (pT2N1b, AJCC 8th edition) was confirmed. Immunostaining showed that the colon cancer was positive for CDX2, SATB2, had a loss of PMS2 and intact expression of MLH1, MSH2 and MSH6, negative for AMACR, while the prostate cancer was positive for AMACR, had intact expression of PMS2, MLH1, MSH2 and MSH6, and negative for CDX2 and SATB2. MSI-H phenotype and PMS2 mutation in the colon cancer were confirmed by microsatellite instability (MSI) PCR and nextgeneration sequencing (NGS), respectively. Through genetic counseling and analysis of the family pedigree, LS was confirmed with colon cancer present in multiple maternal
\end{abstract}

Correspondence to: Jinping Lai, MD, Ph.D., Department of Pathology, Immunology and Laboratory Medicine, University of Florida College of Medicine, Gainesville, FL, 32610, U.S.A. Tel: +13526279240, Fax: +1 3526279242, e-mail: jinpinglai@ufl.edu, or Weidong Liu, e-mail:weidong.liu@csu.edu.cn

Key Words: Lynch syndrome, colorectal carcinoma, prostate cancer; MMR protein, microsatellite instability (MSI), genetic counseling. family members and his brother also had metachronous colon and prostate cancers. To the best of our knowledge, this is the first documented case of synchronous colon and prostate cancers, with isolated PMS2 loss present in the colon cancer while intact DNA mismatch repair (MMR) protein expressions present in the prostate cancer, in the English literature. The pathogenesis, diagnosis and prognosis of this entity are discussed.

Although colorectal carcinoma (CRC) and prostate cancer are two common malignancies in males (1), synchronous primary prostate cancer and $\mathrm{CRC}$ are rarely reported in the English literature. Overall, $2-5 \%$ of the CRCs are seen in patients with Lynch syndrome (LS), that is characterized by clustering of familial colorectal and extracolonic tumors. LS is caused by deleterious mutations in one of the mismatch repair (MMR) genes $M L H 1, M S H 2, M S H 6$, and PMS2. Germline mutations in MMR genes result in carcinomas marked by the presence of microsatellite instability and loss of expression of one or more MMR proteins are the hallmarks of this carcinomas (2, 3). Universal screening for LS using immunohistochemical analysis of the four MMR proteins, coupled with analysis of MLH1 promoter methylation and $B R A F$ mutation for the detection of sporadic MMR deficiency, was recently proposed for all patients newly diagnosed with colorectal cancer (CRC) and adopted in major cancer centers in the USA (4). However, isolated loss of PMS2 expression by immunohistochemistry (IHC) is rarely reported in CRCs, accounting for approximately $4 \%$ of all cases with microsatellite instability (5). The clinical consequences of isolated loss of PMS2 through germline mutations are poorly understood compared to other Lynchassociated MMR gene mutations $(6,7)$.

In this report, we describe a 71-year-old Caucasian male with synchronous prostate carcinoma and CRC with isolated 

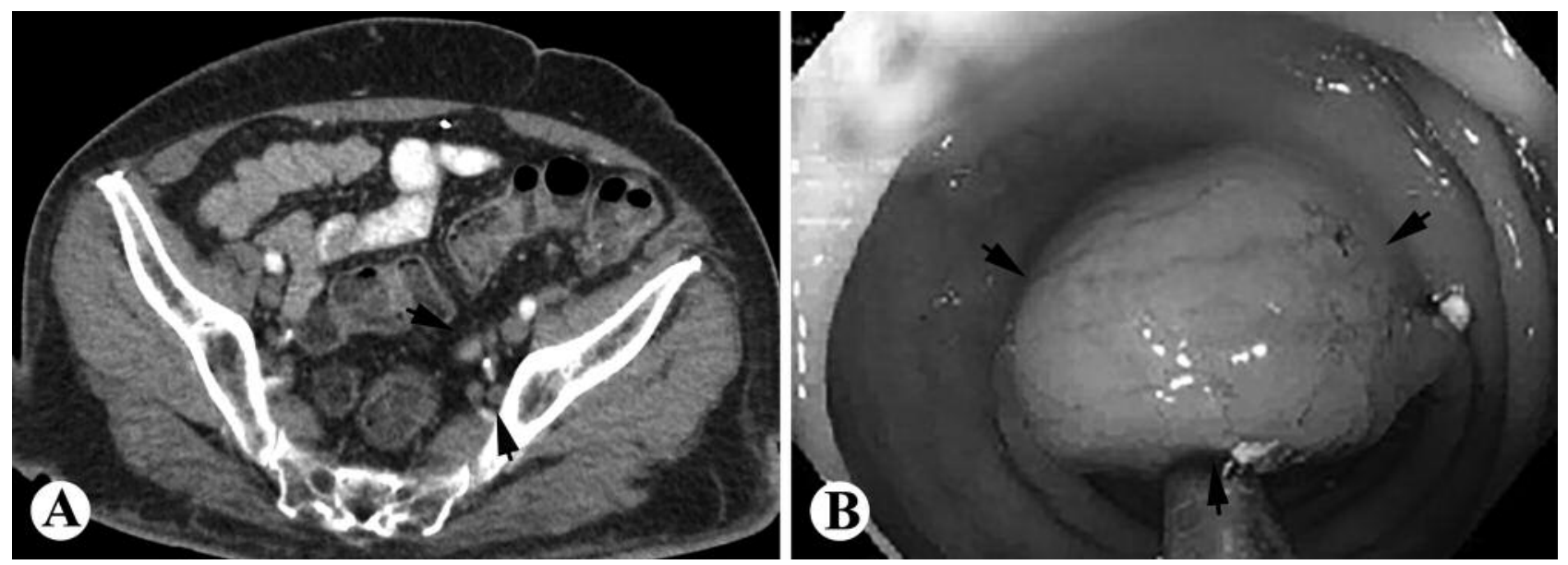

Figure 1. A, Post contrast CT scan showing focal sigmoid colon thickening and adjacent lymphadenopathy (arrows); B, Colonoscopy showing a 3 cm polypoid mass (arrows) present in the sigmoid colon.

loss of PMS2 in his sigmoid colon cancer, but intact PMS2 expression present in his prostate cancer. Genetic counseling and analysis of the family pedigree were performed. The literature was reviewed and the pathogenesis and prognosis of this entity are discussed.

\section{Case Presentation}

A 71-year-old Caucasian male who presented with lower urinary infection treated with Flomax and showed great improvement was referred for prostate biopsy due to a recent increase in the levels of PSA $(10 \mathrm{ng} / \mathrm{ml})$. The patient's medical and social history was significant for cardiac artery disease, diabetes mellitus, hypertension, hyperlipidemia and syncope. The patient had a 40 pack/year smoking history and quit about 37 years ago. His brother and mother had a history of colon carcinoma. His paternal ancestry was Irish and maternal ancestry Portuguese Irish.

Twelve core needle biopsies from lateral base, lateral mid, lateral apex, apex, mid, and base were performed from the bilateral sites of prostate. Two foci involved by prostate adenocarcinoma, Gleason score $7(3+4)$ (grade group 2) involving $10 \%$ of each tissue core were present in the histological diagnosis. The patient was leaning towards considering either robotic prostatectomy or radiation therapy. Workup was performed which would make surgery a better option than radiation. The imaging results from CT scans showed left iliac adenopathy and a possible sigmoid colonic lesion (Figure 1A). A colonoscopy was performed and a 3$\mathrm{cm}$ polypoid lesion was identified in the sigmoid colon (30 $\mathrm{cm}$ from the anal verge) (Figure 1B). The biopsy showed tubulovillous adenoma with high-grade dysplasia and focal area indicative of submucosal invasion. Due to the favorable intermediate prostate adenocarcinoma, the patient was referred to surgical oncology for further treatment of his colon tumor. The patient underwent a laparoscopic anterior resection of the rectosigmoid colon. The specimen was sent to surgical pathology for histology assessment.

\section{Pathology and Molecular Tests}

Grossly, the specimen consisted of a $15 \mathrm{~cm}$ in length and 3 $\mathrm{cm}$ in diameter segment of rectosigmoid colon. Opening of the bowel, there was a $2.9 \times 1.2 \times 0.5 \mathrm{~cm}$ tan-pink, granular tumor with rolled and raised edges. The mesorectum was intact. The mucosa was retracted surrounding the tumor and the tumor appeared to focally involve the muscularis propria. The tumor was $5.9 \mathrm{~cm}$ from the open margin, $7.1 \mathrm{~cm}$ from the stapled margin, $3.5 \mathrm{~cm}$ from the mesenteric margin, and $0.2 \mathrm{~cm}$ from the serosa. Additionally, there were several diverticula identified which were up to $1.1 \mathrm{~cm}$ in the greatest dimension. Sections of the tumor, margins and lymph nodes were submitted for microscopical examination. In histology, the tumor showed low-grade intestinal type of adenocarcinoma with $10 \%$ of mucin components, marked tumor infiltrating lymphocytes and peritumoral Crohn-like lymphocytic response. Two of the 15 lymph nodes were positive for metastatic colon adenocarcinoma. Margins were uninvolved by carcinoma. The colon adenocarcinoma was morphologically different to the adenocarcinoma from the prostate (Figure 2). Immunostaining was performed and the colon tumor cells were positive for CDX2 and SATB2, negative for AMACR, in contrast the tumor cells of the prostate cancer were positive for AMACR and negative for 


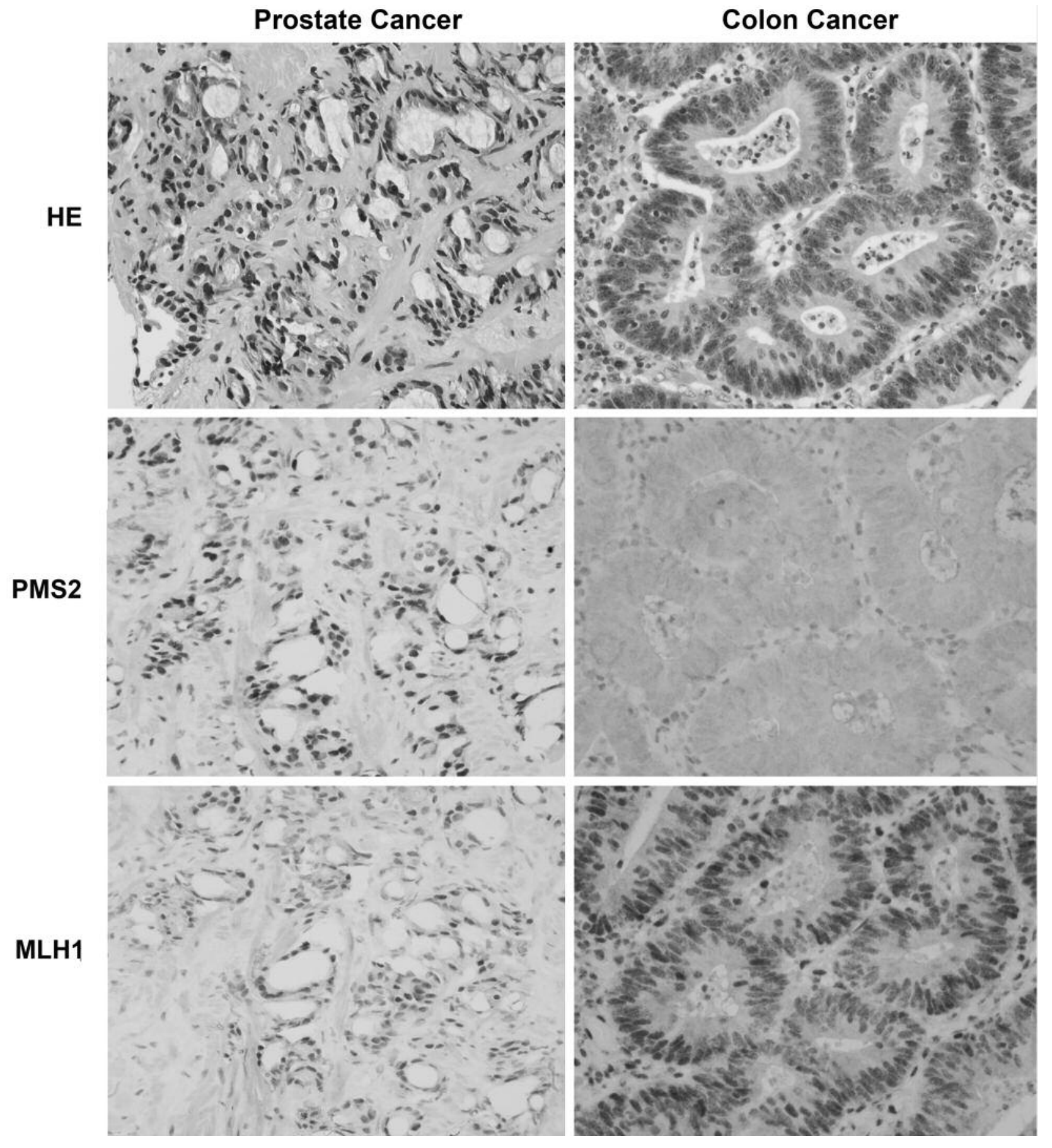

Figure 2. H\&E sections showing the histology of prostate cancer and intestinal type of colonic adenocarcinoma and comparison of the immunoreactivities of PMS2 and MLH1 (all, 400x).

CDX2 and SATB2. The colon adenocarcinoma showed loss of PMS2 and normal expression of MLH1, MSH2 and MSH6, while the prostate cancer showed normal expression of PMS2, MLH1 (Figure 2), MSH2 and MSH6. The final diagnosis of the sigmoid colon tumor was adenocarcinoma, low grade, with a tumor stage of pT2N1b (AJCC 8th Edition) and disease stage IIIA.

Molecular testing of the colon cancer showed that the tumor exhibited microsatellite instability in more than $30 \%$ of the microsatellite repeats tested. MSI was observed with 


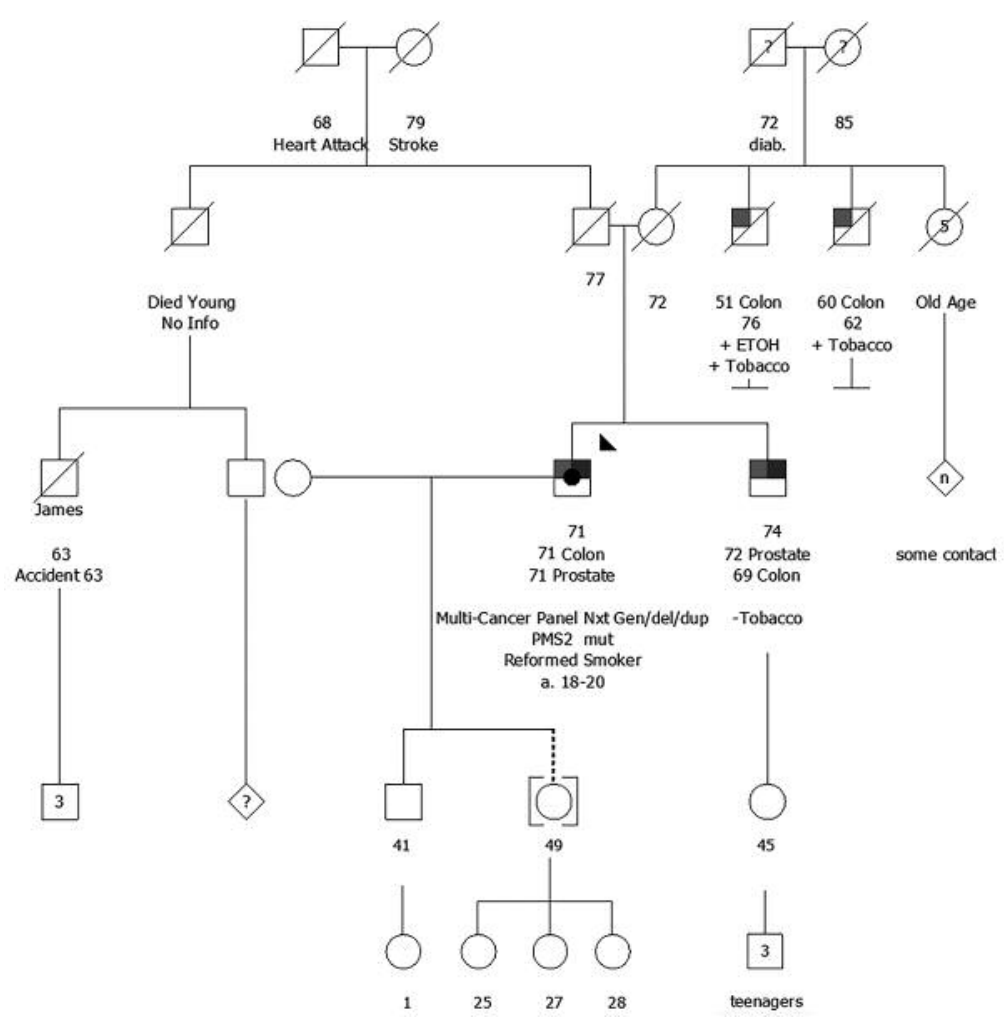

Figure 3. Family pedigree made through cancer gene tests and genetic counseling. The patient had synchronous colon and prostate cancers at 70 years of age, his brother had colon cancer at the age of 69 and prostate cancer at the age of 72, two of his maternal uncles had colon cancer at ages 51 and 60 , respectively.

markers including BAT25, BAT26, NR-21, NR-24, and MONO-27. These results indicate loss of normal DNA mismatch repair function within the tumor.

Next generation sequencing (NGS) was performed in the colon cancer. Mutations in PMS2 (c.7C $>$ T p.Arg3Ter), NRAS (c.182A > G p.Q61R) and TP53 (c.817C >T p.R273C) were present in the cancer cells. Mutations in other genes including NF1, NOTCH1, PHF6, U2AF2, SMAD4, DDR2, $F B X W 7, A L K, A S X L 1$ and $M Y H 11$ were also present.

\section{Follow-up and Genetic Counseling}

The patient recovered well from the surgery and was scheduled for chemotherapy for colon cancer and radiation therapy for his prostate cancer. At the six-month follow up, no significant abnormal imaging findings were identified in his prostate. No lesions or masses were identified in colonoscopy. A biopsy made from the anastomosis showed mild active colitis with no tumor recurrence identified. No metastatic masses were identified in the imaging findings.

The genetic counselling and cancer gene tests including the next generation sequencing and deletion/duplication analysis were performed. Through this analysis, deletion of exon 10 in the PMS2 gene was identified, which is a pathological mutation. Another variant of unknown significance in POLDI c.521G $>$ A (p.Arg174GIn) was also detected. The pathological PMS2 mutation caused the hereditary cancer syndrome LS and increased his chance of developing colorectal cancer at the age of 71 years, and also in his bother at the age of 69 years and his two maternal uncles at the ages of 51 and 60 years respectively (Figure 3 ). His brother also had prostate cancer at the age of 72 years. No carcinomas were identified in the rest of known family members.

\section{Discussion}

Isolated loss of PMS2 expression by IHC is a rare finding in CRCs, accounting for approximately $4 \%$ of all cases with microsatellite instability $(5,8)$. The most common molecular alteration found in such cases is a germline mutation in the $P M S 2$ gene. Lynch syndrome caused by PMS2 mutations is known to have some unusual clinical features (6-8). The patients have an increased risk of developing a variety of different cancers, including colon, endometrial, pancreatic, 
gastric adenocarcinoma, urothelial and thyroid carcinoma. Lynch syndrome patients with an isolated PMS2 mutation not only have a lower lifetime risk of CRC $(\sim 10-20 \%$ by age 70) compared to patients with $M L H 1$ or $M S H 2$ mutations ( $40-50 \%$ by age 70$)$, but the risk of developing extracolonic tumors also appears lower than in patients with mutations in other mismatch repair (MMR) genes (6). In the present case, the patient was diagnosed to have a prostate cancer due to an increase in PSA after treatment of urinary tract infection and his colon cancer was diagnosed during the workup for treatment of prostate cancer at the age of 71 . Colon cancer was present in the family history in multiple family members from his maternal site, identified through genetic counseling and the family pedigree analysis. Prostate cancer was also found in his brother's history, but no other cancers were identified in the family history.

Synchronous cancers are defined as two or more primary cancers diagnosed in the same patient at the same time or within 6 months of each diagnosis $(9,10)$. Our patient is a unique case who had synchronous colon and prostate cancer at the age of 71. Prostate cancer present in Lynch patients is not commonly reported (11). However, a recent study by Kerr et al. showed that in men with Lynch syndrome, 1 of 4 (25\%) was diagnosed to have prostate cancer (12). The index case with Lynch syndrome harbors a heterozygous mutation in the mismatch repair MSH6 gene. Near to complete loss of MSH6 immunoreactivity in the prostate cancer supports silencing of the remaining MSH6 allele during prostate carcinogenesis (12). In our case, MSH6 immunoreactivity was intact. To the best of our knowledge, isolated PMS2 loss in prostate cancer has not been reported in the English literature. In the present case, we found that isolated PMS2 loss was only present in the colon cancer while intact PMS2 expression was present in his prostate cancer. His prostate cancer may not have been associated with Lynch syndrome. Interestingly, the patient's brother also had colon cancer at 69 years of age and prostate cancer at the age of 72 . However, we were not able to obtain the samples from his brother's colon and prostate cancers for MMR IHC/MSI PCR and NGS tests.

Because of the lower likelihood of tumor development in these patients, clinically detecting and managing families with PMS2 mutations has proven difficult $(7,13)$. IHC screening of CRCs for loss of MMR proteins therefore plays an important role in identifying families with PMS2 mutations. The MSI-PCR must be used in the appropriate clinicopathological setting and complemented by immunohistochemistry for mismatch repair (MMR) gene products. Results of this test should be interpreted on the basis of studies published about the influence of MSI on colorectal cancer development and prognosis. MSI is present in the great majority of tumors from patients with hereditary nonpolyposis colorectal cancer (HNPCC). Thus, the absence of MSI makes an HNPCC diagnosis very unlikely. However,
MSI can also occur in patients who do not have HNPCC, but instead have sporadic, non-familial cancer. Approximately $15 \%$ of sporadic colon cancer cases have MSI. In the present case, the morphology of colon cancer showed marked tumor infiltrating lymphocytes, peritumoral Crohn-like lymphocytic response and mucinous components supporting the possibility of MMR/MSI deficient CRC. MMR IHC showed isolated loss of PMS2. However, CRCs with isolated loss of PMS2 expression by IHC could appear to be molecularly heterogeneous. In this case, our MSI also confirmed the MSI-H phenotype and further molecular cancer gene tests by NGS showed the exon 10 PMS 2 mutation. Through a genetic counseling, the patient had Lynch syndrome and colon cancer was present in multiple family members.

Since the clinical consequences of isolated loss of PMS2 are poorly understood as compared with other Lynch-associated MMR gene mutations (6), the exact prognosis is still unknown. In addition, mutations in other genes such as POLD1 were also identified in this case and had unknown clinical significance. Our patient was followed-up for 6 months with no tumor recurrence and metastasis. He is going to have close follow-ups and his family members are aware of this entity for surveillance of colorectal cancer and other associated malignancies.

In summary, we report the first case of Lynch syndrome patient who had synchronous sigmoid colon and prostate cancers at 71 years of age. Our case is unique, as he had isolated loss of PMS2 in the colon cancer while intact expression of PMS2, MSH6, MLH1 and MSH2 in his prostate cancer. MSI-H phenotype and PMS2 mutation were also present in the colon cancer. Genetic counseling revealed that his brother also had colon cancer at the age of 69 and prostate cancer at the age of 72 as well as multiple maternal relatives with a history of colon cancer. The clinical consequences of an isolated PMS2 mutation are so far poorly understood compared to other Lynch-associated MMR gene mutations. Our patient is in regular follow-up and his family members are aware of this entity for surveillance of colorectal cancer and other associated malignancies.

\section{Conflicts of Interest}

The Authors declare that they have no financial conflicts of interest.

\section{References}

1 Duskova K, Vesely S, DO Carmo Silva J, Cernei N, Zitka O, Heger Z, Adam V, Havlova K and Babjuk M: Differences in urinary amino acid patterns in individuals with different types of urological tumor urinary amino acid patterns as markers of urological tumors. In Vivo 32: 425-429, 2018.

2 Boland CR, Koi M, Chang DK and Carethers JM: The biochemical basis of microsatellite instability and abnormal immunohistochemistry and clinical behavior in Lynch syndrome: from bench to bedside. Fam Cancer 7: 41-52, 2008. 
3 Parsons R, Li GM, Longley MJ, Fang WH, Papadopoulos N, Jen J, de la Chapelle A, Kinzler KW, Vogelstein B and Modrich P: Hypermutability and mismatch repair deficiency in RER+ tumor cells. Cell 75: 1227-1236, 1993.

4 National Comprehensive Cancer Network. Genetic/familial highrisk assessment: colorectal (version 2.2016). Available at: www.nccn.org/professionals/physician_gls/pdf/genetics_colon.pd f. Accessed November11, 2016.

5 Dudley B, Brand RE, Thull D, Bahary N, Nikiforova MN and Pai RK: Germline MLH1 mutations are frequently identified in lynch syndrome patients with colorectal and endometrial carcinoma demonstrating isolated loss of PMS2 immunohistochemical expression. Am J Surg Pathol 39: 1114-1120, 2015.

6 ten Broeke SW, Brohet RM, Tops CM, van der Klift HM, Velthuizen ME, Bernstein I, Capellá Munar G2, Gomez Garcia E2, Hoogerbrugge N2, Letteboer TG2, Menko FH2, Lindblom A, Mensenkamp AR, Moller P, van Os TA, Rahner N, Redeker BJ, Sijmons RH, Spruijt L, Suerink M, Vos YJ, Wagner A, Hes FJ, Vasen HF, Nielsen M and Wijnen JT: Lynch syndrome caused by germline PMS2 mutations: delineating the cancer risk. J Clin Oncol 33: 319-325, 2015.

7 Senter L, Clendenning M, Sotamaa K, Hampel H, Green J, Potter JD, Lindblom A, Lagerstedt K, Thibodeau SN, Lindor NM, Young J, Winship I, Dowty JG, White DM, Hopper JL, Baglietto L, Jenkins MA and de la Chapelle A: The clinical phenotype of Lynch syndrome due to germ-line PMS2 mutations. Gastroenterology 135: 419-428, 2008.

8 Gill S, Lindor NM, Burgart LJ, Smalley R, Leontovich O, French AJ, Goldberg RM, Sargent DJ, Jass JR, Hopper JL, Jenkins MA, Young J, Barker MA, Walsh MD, Ruszkiewicz AR and Thibodeau SN: Isolated loss of PMS2 expression in colorectal cancers: frequency, patient age, and familial aggregation. Clin Cancer Res 11: 6466-6471, 2005.
9 Lai JZ, Zhou Y and Cao D: Synchronous pancreatic ductal adenocarcinoma and hepatocellular carcinoma: report of a case and review of the literature. Anticancer Res 38: 3009-3012, 2018.

10 Lai JZ, Lai HH and Cao D: Renal cell carcinoma, unclassified with medullary phenotype and synchronous renal clear cell carcinoma present in a patient with no sickle cell trait/disease: diagnostic and therapeutic challenges. Anticancer Res 38: 37573761, 2018.

11 Paulo P, Maia S, Pinto C, Pinto P, Monteiro A, Peixoto A and Teixeira MR: Targeted next generation sequencing identifies functionally deleterious germline mutations in novel genes in earlyonset/familial prostate cancer. PLoS Genet 14: e1007355, 2018.

12 Kerr L, Rewhorn MJ, Longmuir M, Fraser S, Walsh S, Andrew $\mathrm{N}$ and Leung HY: A cohort analysis of men with a family history of BRCA1/2 and Lynch mutations for prostate cancer. BMC Cancer 16: 529, 2016.

13 Truninger K, Menigatti M, Luz J, Russell A, Haider R, Gebbers JO, Bannwart F, Yurtsever H, Neuweiler J, Riehle HM, Cattaruzza MS, Heinimann K, Schär P, Jiricny J and Marra G: Immunohistochemical analysis reveals high frequency of PMS2 defects in colorectal cancer. Gastroenterology 128: 1160-1171, 2005. 